\title{
Analysis of the Dynamical Behavior of Firms in a Three Layered Modular Assembly Model
}

\author{
${ }^{*}$ Jianjun LU113, Shozo Tokinaga ${ }^{2}$, Wen Tian ${ }^{1}$ \\ ${ }^{1}$ College of Economics and Management, China Agricultural University, Beijing, China \\ ${ }^{2}$ Graduate School of Economics, Kyushu University, Fukuoka, Japan \\ ${ }^{3}$ Australian Maritime College, Launceston, Australia \\ *jlu15@amc.edu.au
}

\begin{abstract}
This paper formulate an option model considers supplier's reaction as the profit sharing in module production, and analyses it by the agent theory. A dynamic environment in the model of the system of the production of modules of three layers is assumed, and the maker and the supplier are modeled by the technique of Genetic Programming (GP) as an agent who takes the action of selfoptimization. As result, the condition that the agent can exist continuously in the market is requested. In conclusion, violent competition and the selection of the similar agent are found even in the model of the option to consider the profit sharing and the reaction.
\end{abstract}

\section{Keywords: Modular Production Scheme; Profit sharing; Reaction Options; agents; Genetic Programming}

\section{Introduction}

Since the introduction of the term supply chain management (SCM) in 1982 (Oliver \& Webber, 1992), it has received ever-growing interest both in the literature as well as from industrial practice (Stadtler, 2005). The supply chain formation between the firms and the module production has been expanded in the background of the upgrade of the information system and the information network (Masuchun, Davis, \& Rangsaritratsamee, 2006, Xiao, Dunne, \& O'Sullivan, 2006, Badr, Biennier, \& Tata, 2011). In approval related to the cooperation between these firms, the effectiveness to which the contract and the relation between dealings are changed according to the change in the situation is discussed (Lu, Kishikawa, \& Tokinaga, 2007, Lu, Tokinaga, \& Ikeda, 2006, Agrawal, Delage, Peters, Wang, \& Ye, 2011, Kannegiesser \& Guenther, 2011). Jaber, Osman, \& Guiffrida (2006) proposed a three-level (supplier-manufacturerretailer) supply chain model with a profit sharing mechanism to maximize the supply chain profit. Increasingly manufacturers and retailers are implementing profit sharing strategies in order to improve channel coordination and supply chain performance. Ruiliang (2008) used a game theory approach to study the strategic role played by profit sharing in a manufacturer-retailer dual-channel supply chain and show that both the manufacturer and the retailer always benefit from a dual-channel profit-sharing strategy because of the incremental profit gains generated by the dual-channel strategy. Yu, Liu, Shen, \& Liu (2007) studied the factors influencing supply chain members' optimal expected profits and the sensitivity to cost information sharing.

Song, Zhang, \& Yang (2009) apply the Closed-loop supply chain coordination management methods to analyses a client enterprise and a third party logistics enterprise's cooperation develop into mature stage in the logistics outsourcing supply chain after the complete information circumstance condition of joint decision, through application of profit sharing contract design to coordinate the supply chain, make both parties can do their best to supply in overall profit maximization, and share common efforts in profits to create a double win outcome. To date, this concept has been formulated as operating flexibility (Kogut \& Kulatilaka, 1994, Huchzermeier \& Loch, 2001 Spinler \& Huchzermeier, 2006 Bendoly, Perry-Smith \& Bachrach, 2010) . It is shown that the model by whom sharing and the reaction of the profit with the firm that becomes the other partner of the contract are taken is introduced, and theoretical results show that there exist equilibrium points among behaviors of makers and suppliers though only the subject that exercises the option is paid attention in the formulation of the operation flexibility (Kamrad \& Siddique, 2004, Hult, Craighead, \& Ketchen, 2010, Martzoukos, 2009, Piccolo \& Reisinger, 2011, Lo-Nigro \& Abbate, 2011). However, these methods postulate the stochastic changes, and it is not clear whether in a more realistic environment the equilibrium is still confirmed. In addition, it should be examined more when a probabilistic model is introduced in such an analysis. 
Moreover, the situation where a rival relation is generated, in which the selection and consolidating are performed, and many suppliers exist stably between the similar makers between suppliers is not seen in present module production (Agrawal et al., 2011, Kannegiesser \& Guenther, 2011) . In this paper, the option model considers supplier's reaction as the profit sharing in such module production as formulated, and it analyses it by the agent theory. A dynamic environment in the model of the system of the production of modules of three layers is assumed, and the maker and the supplier are modeled by the technique of Genetic Programming (GP) as an agent who takes the action of self-optimization. As a result, the condition that the agent can exist continuously in the market is requested. In conclusion, violent competition and the selection of the similar agent are found even in the model of the option to consider the profit sharing and the reaction (Lu et al., 2007, Bernstein \& DeCroix, 2004).

Layer composition in module production: We assume a three layered system of module production. The firm that manufactures the first component is called a supplier, the firm that manufactures the module of the built-in type is called a sub-assembler and the firm that finally assembles all components and sends them off to the market is called an assembler. The produce cost (unit price), prices of component or product modules and the procurement rates of component from upper layers are given respectively the three parts as a variable. The produce cost and the procurement rate are fixed at first, and formulated as an optimization problem. Moreover, it is given how much production capacity has to be thrown out by each subject as a solution.

(Supplier)

$$
\max _{Q_{i j}} \pi_{i j}=-k_{i j} Q_{i j}+\left(\alpha_{i} \gamma_{i j}-c_{i j}\right) \cdot E\left[\min \left(Q^{\min }(Q), D\right)\right]
$$

(Sub-supplier)

$$
\max _{Q_{i}} \pi_{i}=-\left(k_{i 0}+k_{i}\right) Q_{i}+\left(\alpha_{i} \gamma_{i 0}-\left(c_{i 0}+c_{i}\right)\right) \cdot E\left[\min \left(Q^{\min }(Q), D\right)\right]
$$

(Assembler)

$$
\max _{Q_{0}} \pi_{0}=-k_{0} Q_{0}+\left(\alpha_{0} \gamma_{0}+\left(\alpha_{0}-c_{0}\right)\right) \cdot E\left[\min \left(Q^{\min }(Q), D\right)\right]
$$

Where $\pi$ is profit, $\gamma, c, k$ is payment ratio to lower-rank members respectively, $c, k$ is unit cost and unit capacity cost for component production for a high-ranking member. $Q$ Is selected production capacity.

\section{Fig 1: Model of three layered module production system}

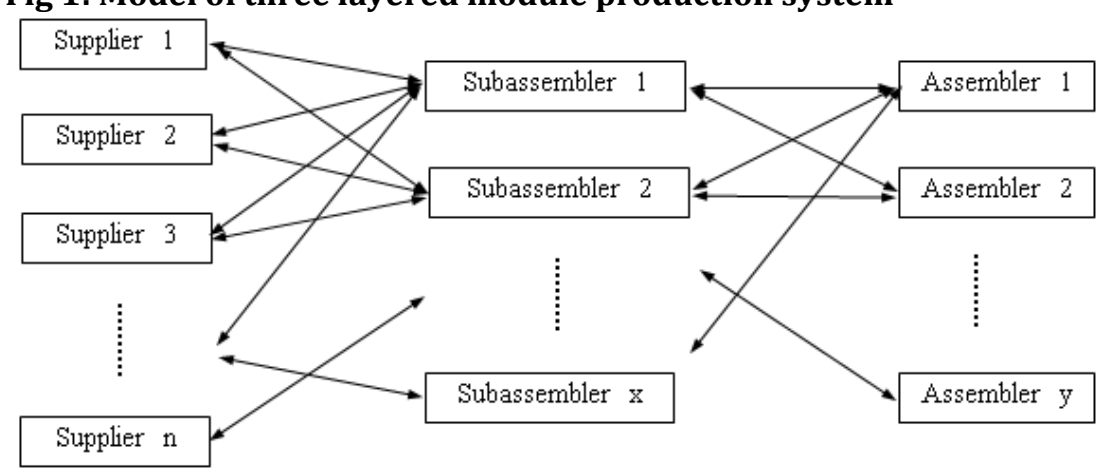

The problem is solved by the method of newsvendor problem (Petruzzi \& Dada, 1999) (Agrawal \& Seshadri, 2000) (Schweitzer \& Cachon, 2000) (Dada, Petruzzi, \& Schwarz, 2007; Yue, Chen, \& Wang, 2006) (Yan, Ruoxuan, Vakharia, Yuwen, \& Seref, 2011). The newsvendor model is a mathematical model in operations management and applied economics used to determine optimal inventory levels. It is typically characterized by fixed prices and uncertain demand for a perishable product. If the inventory level is q, each unit of demand above $\mathrm{q}$ is lost. This model is also known as the Newsvendor Problem or Newsboy Problem by analogy with the situation faced by a newspaper vendor who must decide how many copies of the day's paper to stock in the face of uncertain demand and knowing that unsold copies will be worthless at the end of the day (Stevenson, 2008). In the following, we show the model when the produce cost and the purchase rate are changed. Furthermore, we illustrate the method of deciding the best produce cost and the purchase rate in each subject. However, the solution has to solve only one subject for each subject is homogeneous. Therefore, we show only the first subject as form of solution. 


\section{Model of three layered production system}

This differs from the static evaluations. However, recently, the problem instituted by Kamrad and Siddique is a model, the side of the structure and the supplier who reduces the profit to both the maker and the supplier by whom the option is given(Kamrad \& Siddique, 2004) (Katok \& Wu, 2009). Kamrad and Siddique (2004) modeled how flexibility can be mutually beneficial to both the producer and the suppliers using a real-options (contingent claims) approach, analyze and value supply contracts in a setting characterized by exchange rate uncertainty, supplier-switching options, order-quantity flexibility, profit sharing, and supplier reaction options. The research concurrent solves and examine the dual optimization problem for the suppliers and the producer and endogamies the extent and degree of profit sharing through the resulting optimal policies. In a current discussion, the model where there is leadership in the maker, and by whom the supplier's selection and factory location can be one-sidedly selected, is assumed. However, the maker's excessive demand can be refused, or the place for comparative study with another maker has certainly been left even on a supplier's side. That is, the model where the structure of win-win among members who form the supply chain is guaranteed is needed.

In consideration of the above, in this paper, we propose the model in which the production version of three layers is assumed. Each composition subject self-optimizes the action based on GP, and the feature is analyzed by the simulation. Hence, when procurement ratio $u_{i}$ from the supplier decreases, margin $C_{i}\left(u_{i}\right)$ in inverse proportion to this is paid. Function $C_{i}(u)$ is assumed a monotone decreasing for variable $\left(C_{i}^{\prime}(u)<0\right)^{u}$.

First, it is necessary to explain the model.

Sub-assembler and supplier: A sub-assembler can have dealings over any supplier, and uses supplier's ability for the other partner's selection. Concretely, the number of matches the next time is forecast by using a supplier's present property profit and the number of matches until the last time. This forecast type is described by the arithmetic expression that uses the variable given, and improved by GP. The supplier's reliability and ability can be reflected by the forecast values of the number of matches, for the number are numerical values from how much sub-assembler this supplier has received the demand of procurement. The sub-assembler procures components in proportion to the forecast values of the number of matches obtained about the supplier. The sub-assembler reduces the profit to the supplier in proportion to the ratio in which components are procured. At the same time, the cost to assemble the module is borne. In the relation between the sub-assembler and the supplier, the agent's reaction is put in as a model. Moreover, the supplier $i$ to whom the procurement ratio was lowered receives the margin $C_{i}\left(u_{i}\right)$ proportional to the reduced size from the sub-assembler. As a result, the correction related to the contract from a one-sided sub-assembler can be controlled.

Sub-assembler and assembler: The assembler finally completes a product purchase module from the sub-assembler, and does the work built in. Therefore, it is solved in this case; the sub-assembler's and the supplier's similar contract relation is assumed though procurement from the sub-assembler is executed. The sub-assembler forecasts the match number at the next time by using a present property profit and the match number until the last time. This forecast equation is described by the arithmetic expression that uses the variable given, and improves it by GP. The sub-assembler 's reliability and ability can be reflected by the forecast values of the match number, for the number are numerical values that show how much the supplier has received purchase demand from the sub-assembler. The assembler purchases components in proportion to the forecast of the match number obtained from the sub-assembler. The assembler reduces the profit to the sub-assembler in proportion to the ratio the components are purchased and, at the same time, he bears the cost to assemble the module. In the relation between the assembler and the sub-supplier, the agent's reaction is put in as a model. The sub-assembler to which the procurement ratio is lowered receives margin $C_{i}$ from the assembler

GP and Modeling agent system: The method of GP: In the following, we describe the outline of GP only (there are many descriptions about modeling the agent study by GP(J. Lu et al., 2006) (Lu et al., 2007) . GP is an extension of the conventional Genetic Algrithm (GA) in which each individual in the population (pool of individuals) is a computer program composed of arithmetic operations, standard mathematical expressions, and variables. For the sake of simplicity, we will demonstrate the GP procedure to use to 
approximate functions when predicting the time series. In GP, the system equations (called "individuals") are represented in the tree structure. In the parse tree, non-terminal nodes are taken from well-defined functions, such as binomial operation,,$+- \times, \div$, and the operation takes the square root of the variable. For example, in time series prediction, the terminal nodes consist of arguments chosen from a set of constant variables, such as $x(t-1)$, which is the time lag of $x(t)$. Usually, we calculate the root mean square error between $x(t)$ and $\tilde{x}(t)$, where $\tilde{x}(t)$ the prediction of is $x(t)$ obtained by an individual, and use it to define fitness. In using the measure of fitness to evaluate each individual, we applied GP to the population to derive better descriptions of the future actions that guarantee higher profits. Because we selected a pair of individuals with higher fitness levels, the crossover operation could be applied to generate new individuals. Two sub trees from a pair of individuals were extracted and swapped with each other.

To ensure that the crossover operation produced syntactically and semantically valid programs, we looked for nodes that could serve as sub trees in the crossover operation and confirmed that there were no violations. The crossover operation created new offspring by exchanging sub-trees between two parents. We also used mutation operations. The goal of the mutation operations was to reintroduce some diversity into the population. Two types of GP mutation operations were utilized to replace a part of the tree with another element.

Global mutation: G-mutation: We generated individual $I$, and selected a sub tree with consistent representation. Then, we selected a sub tree from the individual $J$ pool at random, and replaced the sub tree with the sub tree of individual $I$. After the mutation, we only retained the modified individual $J$ in the pool.

Local mutation: L-mutation: We then randomly selected a locus in parse tree $J$ to which the mutation was applied. We replaced the place with another value (a primitive function or a variable). We iteratively performed the following steps until the termination criterion had been satisfied.

- Step 1: Generate an initial, randomly composed population of possible functions and terminals for the problem at hand. The random tree must be from a syntactically correct program.

- Step 2: Execute each individual (evaluation of system equation) in the population, and assign it a fitness value, giving partial credit for getting close to the correct output. Then, sort the individuals according to their fitness $\mathrm{Si}$.

- Step 3: Select a pair of individuals with a probability pi, based on their fitness levels. The probability $p i$ is defined for $i$ th individual as follows:

$p_{i}=\left(S_{i}-S_{\min }\right) / \sum^{n}\left(S_{i}-S_{\min }\right)$

Where $S_{\min }$ the minimum is value of $S_{i}$, and $N$ is the population size.

- Step 4: Then, create new individuals (offspring) from the selected pair by genetically recombining the randomly chosen parts of two existing individuals with the crossover operation, which should be applied at a randomly selected crossover point.

- Step 5: At a certain probability, we apply the mutation operations to the pool of individuals. If the result designation is obtained by the GP (i.e., the maximum value of the fitness becomes larger than the prescribed value), terminate the algorithm. Otherwise, go to Step 2.

Modeling agent system based on GP: The agent calculates the score evaluation value in order to select the agent, who becomes the other partner of dealings by the arithmetic expression advantageously. Two or more calculation types exist, and the calculation type uses the most excellent forecast type from a past forecast performance. The sub-assembler forecasts the number of matches of suppliers for the next time. The assembler similarly forecasts the number of matches of sub-assemblers. GP is used for the forecast improvement of this function. As a result, the best production at the next time is carried out. The agent has sets of the forecast types (this is called an individual pool), and uses the GP for executing inherited intersection processing, etc. as a method of improving this performance as shown in Fig.2. 
Fig 2: Description that uses agent theory

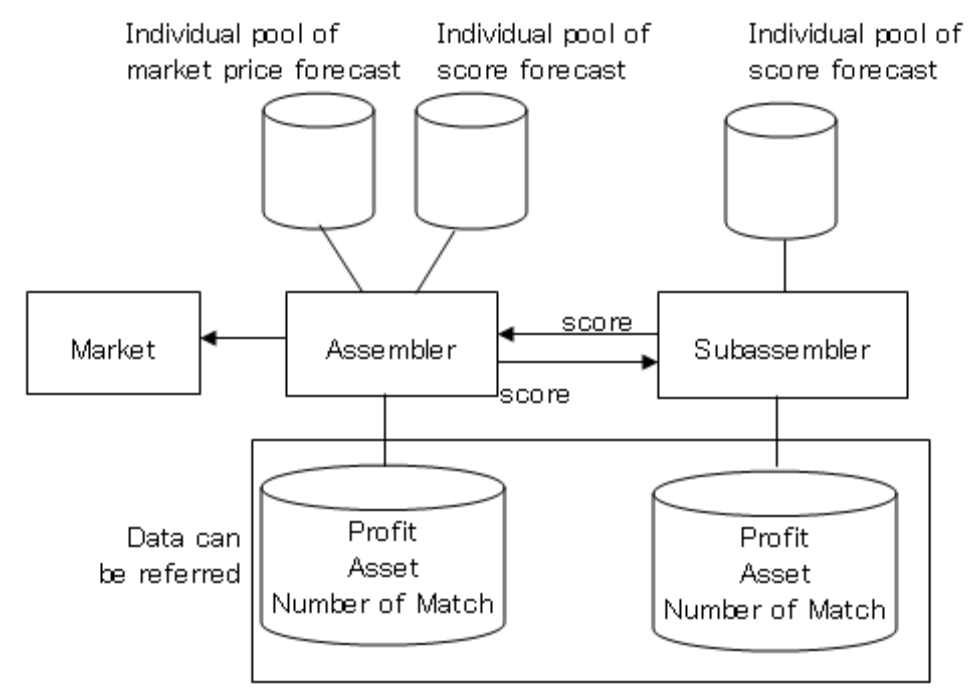

Application: In the following, the character of the profit that each agent firm of the three layered acquires is analyzed by the simulation. The model of the simulation is set as follows:

Number of agents: 100

Size of individual pool: 1000

The method of distributing the profit: part of the sales profit of the assembler in the market is reduced to the sub-assembler, and its part is reduced further by the supplier. This relates to the module production, and whether the following is found in analysis.

1) Profit accumulation by sub-assembler: It is easy to become a Black Box even if it sees from others which agents because the sub-assembler is located with the supplier between assemblers. Therefore, there are price fluctuations of component (when decreasing chiefly) and there is danger not reflected in the cost reduction in the assembler. The profit of the sub-assembler presumed by the agent system is put as $r_{s}$. In order to analyze the action of such a sub-assembler, the profit of the sub-assembler when the supplier cannot be selected is presumed and the profit is given as $r_{w}$ (sub-assembler does not have enough forecast ability). The difference of presumption $r_{s}, r_{w}$ of these two profits becomes the accumulation of profit by the sub-assembler. When some parameters are set, an average profit increase of these cases is shown in Fig. 3 and 4. It is seen from the figure that the profit decreases when the forecast of prices of the component of the sub-assembler is not accurate. Thus, the situation of the profit accumulation in the sub-assembler that is, making to the Black Box is confirmed.

\section{Fig 3: Profit increase of accurate price forecast of sub-assembler}

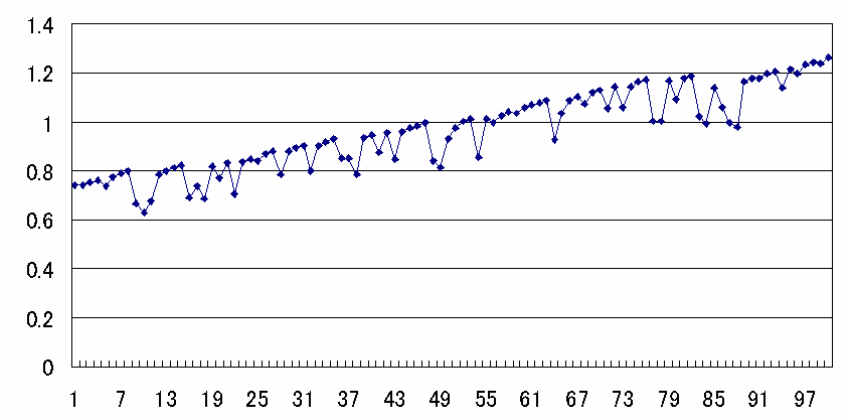


Fig 4: Profit increase of inaccurate price forecast of sub-assembler

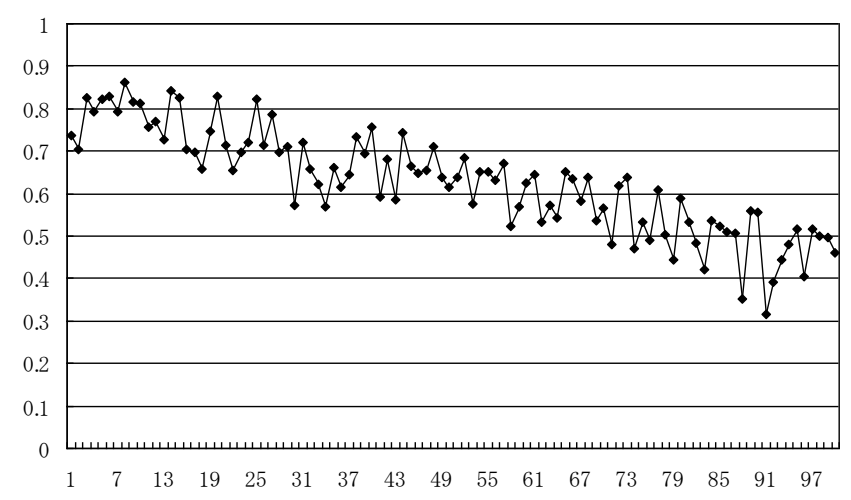

2) Selection of sub-assembler: A sub assembler not so examines whether to be weeded out though the sub-assembler adequately answered can be survived the competition the demand from the assembler. It is possible to correspond to the profit acquisition from the supplier, and the selection happens when the forecast of the score that controls the selection and the profit sharing of the assembler are not correct. When the number of sub-assemblers to which the assembler matches is limited, a remarkable tendency is found. This is common with the phenomenon seen in the two layered production system that authors have demonstrated.

3) Vibration of affiliations: In the time series of maker's profit, a chaos vibration is observed. A chaos vibration is similarly observed in the time series of the margin of the supplier. The change in the number of matches of agents when the allowable number that can be comparatively matched is greatly taken is shown in Fig.5. Thus, when the other partner firm that can select it is made gradual, the other partner agent is not easily limited. However, when the number of matches that can be allowed is limited, the number of matches omits a steady result.

\section{Fig 5: Example of change in match number}

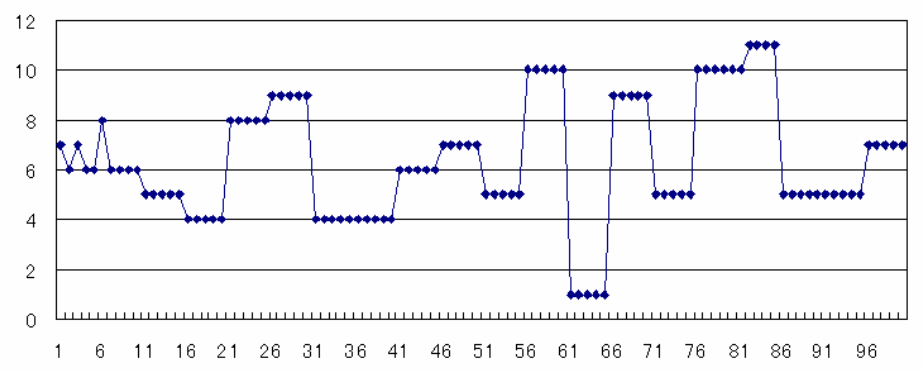

\section{Discussion and Conclusion}

In this paper, to build a win-win structure among members of supply chain, we analyzed the dynamical behavior of firms in three layered modular assembly models including the profit sharing and reaction of firms. This model considering the maker's excessive demand and the place for comparative study with another maker has certainly been left even on a supplier's side. The firms are regarded as agents who make decisions based on the predictions obtained by Genetic Programming. Rather than Genetic Algrithm (GA), In GP, non-terminal nodes in the parse tree can be taken from well-defined functions, such as binomial operation,,$+- \times, \div$, and the operation takes the square root of the variable. The agent calculates the score evaluation value in order to select the agent, who becomes the other partner of dealings by the arithmetic expression advantageously. Two or more calculation types exist, and the calculation type uses the most excellent forecast type from a past forecast performance. The sub-assembler forecasts the number of matches of suppliers for the next time. The assembler similarly forecasts the number of matches of sub-assemblers. GP is used for the forecast improvement of this function. As a result, the best production at the next time is carried out. Based on the profit distributing method that part of the sales profit of the assembler in the market is reduced to the sub-assembler, and its part is reduced further by the supplier, simulate the character of the profit that each agent firm of the three layered acquires. By 
using the simulation studies, this revealed that even in the profit sharing and reaction option models, there is weeding out among firms and chaotic behavior in increases of profits.

\section{References}

Agrawal, S., Delage, E., Peters, M., Wang, Z. \& Ye, Y. (2011). A Unified Framework for Dynamic Prediction Market Design. Operations Research, 59(3), 550-568.

Agrawal, V. \& Seshadri, S. (2000). Impact of uncertainty and risk aversion on price and order quantity in the newsvendor problem. Manufacturing \& Service Operations Management, 2(4), 410-422422.

Badr, Y., Biennier, F. \& Tata, S. (2011). The integration of corporate security strategies in collaborative business processes. IEEE Transactions on Services Computing, 4(3), 243-254254.

Bendoly, E., Perry-Smith, J. E. \& Bachrach, D. G. (2010). The perception of difficulty in project-work planning and its impact on resource sharing. Journal of Operations Management, 28(5), 385-397.

Bernstein, F. \& DeCroix, G. A. (2004). Decentralized pricing and capacity decisions in a multitier system with modular assembly. Management Science, 50(9), 1293-1308.

Dada, M., Petruzzi, N. C. \& Schwarz, L. B. (2007). A newsvendor's procurement problem when suppliers are unreliable. [Article]. M\&Som-Manufacturing \& Service Operations Management, 9(1), 9-32.

Huchzermeier, A. \& Loch, C. H. (2001). Project management under risk: Using the real options approach to evaluate flexibility in R\&D. Management Science, 47(1), 85-101.

Hult, G. T. M., Craighead, C. W. \& Ketchen, D. J. (2010). Risk Uncertainty and Supply Chain Decisions: A Real Options Perspective. Decision Sciences, 41(3), 435-458.

Jaber, M. Y., Osman, I. H. \& Guiffrida, A. L. (2006). Coordinating a three-level supply chain with price discounts, price dependent demand, and profit sharing. International Journal of Integrated Supply Management, 2(1-2), 28-48.

Kamrad, B. \& Siddique, A. (2004). Supply contracts, profit sharing, switching, and reaction options. Management Science, 50(1), 64-82.

Kannegiesser, M. \& Guenther, H. O. (2011). An integrated optimization model for managing the global value chain of a chemical commodities manufacturer. Journal of the Operational Research Society, 62(4), 711-721.

Katok, E. \& Wu, D. Y. (2009). Contracting in Supply Chains: A Laboratory Investigation. [Article]. Management Science, 55(12), 1953-1968.

Kogut, B. \& Kulatilaka, N. (1994). Operating Flexibility, Global Manufacturing, and The Option Value of a Multinational Network. Management Science, 40(1), 123-139.

Lo-Nigro, G. \& Abbate, L. (2011). Risk assessment and profit sharing in business networks. International Journal of Production Economics, 131(1), 234-241.

Lu, J., Kishikawa, Y. \& Tokinaga, S. (2007). Realization of feature description systems for clusters by rule generation based on genetic programming and its applications. Electronics and Communications in Japan Part Ii-Electronics, 90(9), 87-97.

Lu, J., Tokinaga, S. \& Ikeda, Y. (2006). Explanatory rule extraction based on the trained neural network and the genetic programming. Journal of the Operations Research Society of Japan, 49(1), 66-82.

Martzoukos, S. H. (2009). Real R\&D options and optimal activation of two-dimensional random controls. Journal of the Operational Research Society, 60(6), 843-858.

Masuchun, W., Davis, S. \& Rangsaritratsamee, R. (2006). System for supply network management. Information Technology \& Management, 7(4), 315-333.

Oliver, R. K. \& Webber, M. D. (1992). Supply-chain management: Logistics catches up with strategy (reprint from Outlook 1982). In M. Christopher (Ed.), Logistics : the strategic issues (63-75). London.

Petruzzi, N. C. \& Dada, M. (1999). Pricing and the newsvendor problem: A review with extensions. [Article]. Operations Research, 47(2), 183-194.

Piccolo, S. \& Reisinger, M. (2011). Exclusive Territories and Manufacturers' Collusion. Management Science, 57(7), 1250-1266.

Ruiliang, Y. (2008). Profit sharing and firm performance in the manufacturer-retailer dual-channel supply chain. Electronic Commerce Research, 8(3), 155-172.

Schweitzer, M. E. \& Cachon, G. P. (2000). Decision bias in the newsvendor problem with a known demand distribution: Experimental evidence. [Article]. Management Science, 46(3), 404-420.

Song, Z. J., Zhang, Y. \& Yang, J. M. (2009). Closed-loop Outsourcing Logistics Supply Chain Coordination Management Based on the Profit-sharing Contract. New York: Ieee.

Spinler, S. \& Huchzermeier, A. (2006). The valuation of options on capacity with cost and demand uncertainty. European Journal of Operational Research, 171(3), 915-934. 
Stadtler, H. (2005). Supply chain management and advanced planning - basics, overview and challenges. [Review]. European Journal of Operational Research, 163(3), 575-588.

Stevenson, W. (2008). Operations Management (10 edition ed.): McGraw-Hill/Irwin.

Xiao, G., Dunne, K. \& O'Sullivan, D. (2006). Development of an online supplier selection module. International Journal of Services Technology and Management, 7(1), 52-6767.

Yan, Q., Ruoxuan, W., Vakharia, A. J., Yuwen, C. \& Seref, M. M. H. (2011). The newsvendor problem: Review and directions for future research. European Journal of Operational Research, 213(2), 361-374.

Yu, R., Liu, L., Shen, Y. \& Liu, L. (2007). Factors influencing supply chain members profits with cost information sharing. Journal of Tsinghua University (Science and Technology), 47(5), 749-752.

Yue, J. F., Chen, B. T. \& Wang, M. C. (2006). Expected value of distribution information for the newsvendor problem. [Article]. Operations Research, 54(6), 1128-1136. 